\title{
A STUDY OF R-MAGNITUDE DEPENDENCE IN SPATIAL ORIENTATION OF SPIN VECTORS OF SDSS DR-7 GALAXIES OF REDSHIFT $0.10<\mathrm{Z}<0.11$
}

\author{
Shiv Narayan Yadav \\ Patan Multiple Campus, Patan, Tribhuvan University, Lalitpur, Nepal.
}

\begin{abstract}
Abtract: We present a study of spin vector orientations of 44749 r-magnitude SDSS (Sloan Digital Sky Survey) galaxies having redshift 0.10 to 0.11 . The $\mathrm{r}$-magnitudes are observed through $616.5 \mathrm{~nm}$ CCD (charge coupled device) filter attached to SDSS telescope located at New Mexico, USA. We have converted two dimensional data to three dimensional galaxy rotation axes. Our aim is to find out non-random effects in the spatial orientation of galaxies. In addition, we wanted to check r-magnitude dependence in the spatial orientation. The expected isotropy distribution curves are obtained by removing the selection effects and performing a random simulation method. In general, spin vector orientations of galaxies is found to be random, supporting Hierarchy model of galaxy formation. A local anisotropy is observed in few samples suggesting a gravitational tidal interaction between neighbor galaxies.
\end{abstract}

Keywords: Super clusters; Galaxies; Clusters; Evolution; Statistics.

\section{INTRODUCTION}

Galaxy clusters are gravitationally bound large scale structures in the universe. To understand the evolution of these aggregates it is essential to know when and how they have formed and how their structures and constituents have been changing with time. Gamow made it clear that the observed rotations of galaxies are important for cosmology ${ }^{1}$. According to them, the fact that galaxies rotate might be a clue of physical conditions under which these systems formed. Thus, understanding the distribution of spatial orientations of the spin vectors (hereafter SVs) or angular momentum vectors of galaxies is very important. It could allow us to know the origin of angular momenta of galaxies.

There are three predictions about the spatial orientation of spin vectors of galaxies. These are the 'pancake model', the 'hierarchy model,' and the 'primordial vorticity theory.' The 'pancake model' predicts that the spin vectors (SVs hereafter) of galaxies tend to lie within the cluster plane ${ }^{2,3,4}$. According to the 'hierarchy model', the directions of the SVs should be distributed randomly ${ }^{5}$. The 'primordial vorticity theory' predicts that the spin vectors of galaxies are distributed primarily perpendicular to the cluster plane ${ }^{6,7}$.In the present work we intend to work on SDSS ( $7^{\text {th }}$ data release) database of galaxies that have redshift $0.10<z<0.11$. We are interested to check whether magnitude dependence exist concerning galaxy orientation or not.

\section{Godlowskian transformation}

The three dimensional orientation of the SV of a galaxy is characterized by two angles: the polar angle $(\theta)$ between the galactic SV and a reference plane (here equatorial plane), and the azimuthal angle $(\Phi)$ between the projection of a galactic SV on to this reference plane and the $\mathrm{X}$-axis within this plane. The detail derivations of the expressions of the angles $\theta$ and $\Phi^{8}$. Using equatorial coordinate system as reference, then $\theta$ and $\Phi$ can be obtained from measurable quantities as follows:

$\sin \theta=-\cos i \sin \alpha \pm \sin i \sin P \cos \delta$

$$
\begin{array}{r}
\operatorname{Sin} \phi=(\cos \theta)^{-1}[-\cos i \cos \delta \sin \alpha+\sin i(\overline{+} \sin P \sin \delta \sin \alpha \mp \\
\cos P \cos \alpha)]
\end{array}
$$

Where, $I$ is the inclination angle, the angle between the normal to the galaxy plane and the observer's line-ofsight. The inclination angle can be computed from the formula,

$\cos ^{2} i=\frac{\left(q^{2}-q^{*^{2}}\right)}{\left(1-q^{*^{2}}\right)}$

This expression is valid for oblate spheroids ${ }^{9}$. Here, $q$ and $q^{*}$ represent the measured axial ratio $(b / a)$ and the intrinsic flatness of the galaxy, respectively. The intrinsic flatness of a disk galaxy depends on the morphological type ${ }^{10}$. Hiedmann showed that the values of $q^{*}$ range from 0.083 for Sd spirals to 0.33 for ellipticals. For the galaxies with unknown morphology $q^{*}=0.20$ is assumed ${ }^{10}$.

\section{Numerical simulation}

Galaxies for respective parameters. We use these numbers to make input file and the expected distribution Here we describe the procedure for the removal the selection effects to obtain the isotropic distributions for both $\theta$ and $\Phi^{11}$. Theoretically, the isotropic distribution curve for polar angle is cosine and that for azimuthal angle is the average distribution curve, with the restriction that the database is free from se lection effect. Aryal\&Saurer concluded that any selections imposed on the database may cause severe changes in the shapes of the expected isotropic distribution curves ${ }^{[11]}$. In their method, a true spatial distribution of the galaxy rotation axis is assumed to be isotropic. Then, due to the projection effects, $i$ can be distributed as $\sim \sin i, B$ can be distributed $\cos B$, the variables $\alpha$ and $P$ can be distributed randomly, and the equation $(1,2)$ can be used to calculate the corresponding values of polar $(\theta)$ and azimuthal $(\Phi)$. We run simulations in order to define expected isotropic distribution curves for both the $\theta$ and $\Phi$ distributions. The isotropic distribution curves are based on simulations including $10^{7}$ virtual galaxies. At first we observed the distributions of 
$\alpha, \delta, \mathrm{P}$ and $\mathrm{i}$ for the galaxies in our samples and distributed by creating $10^{7}$ virtual by running simulation in MATLAB 7.0.

\section{METHOD OF ANALYSIS}

Our observations (real observed dataset) are compared with the isotropic distribution curves (obtained from simulation) in both $\theta$ and $\Phi$ distributions. For this comparison we use three different statistical tests: chisquare-, Fourier-, and auto correlation-test.

\section{Chi-square test}

The $\chi^{2}$ test is a measure of how well a theoretical probability distribution fits a set of data. The test is an objective way to examine whether the observational distribution deviates from the expected distribution (in our case, to the isotropic distribution), which is based on the reduced $x^{2}$ value given by,

$\chi_{v}^{2}=\frac{\chi^{2}}{v}$

$\chi^{2}=\sum_{i=1}^{n} \frac{\left(N_{0 i}-N_{e i}\right)^{2}}{N_{e i}}$

Here, $n$ represents the number of bins, $N_{0 i}$ and $N_{e i}$ represent the observed and expected isotropic distributions and $v$ is the degree of freedom $(v=n-1)$. For an isotropic distribution, the $\chi_{v}^{2}$ value is expected to be nearly zero. The quantity $\mathrm{P}\left(>\chi_{v}^{2}\right)$ gives the probability that the observed $\chi_{v}^{2}$ value is realised by the isotropic distribution. The observed distribution is more consistent with the expected isotropic distribution when $\mathrm{P}\left(>\chi_{v}^{2}\right)$ is larger. We set $\mathrm{P}\left(>\chi_{v}^{2}\right)=0.050$ as the critical value to discriminate isotropy from anisotropy, it corresponds to the deviation from the isotropy at $2 \sigma$ level.

\section{Fourier test}

If the deviation from isotropy is a slowly varying function of the angle $\theta$ (or $\Phi$ ), one can use the Fourier test. Let $N$ denote the total number of solutions for galaxies in the sample, $N_{k}$ the number of solutions in the $k^{\text {th }}$ bin, $N_{0}$ the mean number of solutions per bin, and $N_{0 k}$ the expected number of solutions in the $k^{\text {th }}$ bin. Then the Fourier series becomes,

$N_{k}=N_{0}\left(1+\Delta_{11} \cos 2 \theta_{k}+\Delta_{21} \sin 2 \theta_{k}+\Delta_{12} \cos 4 \theta_{k}+\Delta_{22} \sin 4 \theta_{k}+\cdots\right)$

Here the angle $\theta_{k}$ represents the polar angle in the $k$ th bin. Taking first order Fourier mode,

$N_{k}=N_{0}\left(1+\Delta_{11} \cos 2 \theta_{k}+\Delta_{21} \sin 2 \theta_{k}\right)$

Here the Fourier coefficients $\Delta_{11}$ and $\Delta_{21}$ are the parameters of the distributions. We obtain the following expressions for the Fourier coefficients $\Delta_{11}$ and $\Delta_{21}$,

$$
\begin{aligned}
& \Delta_{11}=\frac{\sum_{k=1}^{n}\left(N_{k}-N_{0 k}\right) \cos 2 \theta_{k}}{\sum_{k=1}^{n} N_{0 k} \cos ^{2} 2 \theta_{k}} \\
& \Delta_{21}=\frac{\sum_{k=1}^{n}\left(N_{k}-N_{0 k}\right) \sin 2 \theta_{k}}{\sum_{k=1}^{n} N_{0 k} \sin ^{2} 2 \theta_{k}}
\end{aligned}
$$

The standard deviations $\sigma\left(\Delta_{11}\right)$ and $\sigma\left(\Delta_{21}\right)$ can be obtained using the expressions,

$$
\begin{aligned}
& \sigma\left(\Delta_{11}\right)=\left(\sum_{k=1}^{n} N_{0 k} \cos ^{2} 2 \theta_{k}\right)^{-1 / 2} \\
& \sigma\left(\Delta_{21}\right)=\left(\sum_{k=1}^{n} N_{0 k} \sin ^{2} 2 \theta_{k}\right)^{-1 / 2}
\end{aligned}
$$

The probability that the amplitude,

$\Delta_{1}=\left(\Delta_{11}^{2}+\Delta_{21}^{2}\right)^{1 / 2}$

is greater than a certain chosen value is given by the formula,

$P\left(>\Delta_{1}\right)=\exp \left(-\frac{n}{4} N_{0} \Delta_{1}^{2}\right)$,

With standard deviation,

$\sigma\left(\Delta_{1}\right)=\left(\frac{2}{n N_{0}}\right)^{1 / 2}$

The Fourier coefficient $\Delta_{11}$ is very important because it gives the direction of departure from isotropy.

\section{Auto correlation test}

Auto correlation test is a measure of a degree to which there is a linear relationship between two variables. In our case, it takes account the correlation between the number of galaxies in adjoining angular bins. The correlation function is,

$C=\sum_{1}^{n} \frac{\left(N_{k}-N_{0 k}\right)\left(N_{k+1}-N_{0 k+1}\right)}{\left(N_{0 k} N_{0 k+1}\right)^{1 / 2}}$

with the standard deviation,

$\sigma(C)=(n)^{1 / 2}$

In an isotropic distribution any correlation vanishes, so we expect to have $C \rightarrow 0$.

\section{RESULTS AND DISCUSSION}

Any deviation from expected isotropic distribution will be tested using four statistical parameters, namely chi-square probability $\left(\mathrm{P}>\chi^{2}\right)$, autocorrelation coefficient $(\mathrm{C} / \mathrm{C}(\sigma))$, first order Fourier coefficient $\left(\Delta_{11} / \sigma\left(\Delta_{11}\right)\right)$ and first order Fourier probability $\left(\mathrm{P}>\Delta_{1}\right)$. For anisotropy, the limit of chi-square probability $\mathrm{P}\left(>\chi^{2}\right)$ is $<0.050$, auto correlation coefficient $(\mathrm{C} / \mathrm{C}(\sigma))$ is $>1.0$, first order Fourier 
coefficient $\left(\Delta_{11} / \sigma\left(\Delta_{11}\right)\right)$ is $>1.5$ and Fourier probability $\mathrm{P}\left(>\Delta_{1}\right)$ is $<0.150$ respectively. Any 'hump' (more solutions than the expected) or 'dip' (less solutions than the expected) in the histogram will be discussed as a local effect in the samples. The statistics for the polar and azimuthal angle distributions is given in Table 1. In the statistics of $\theta$, a negative value of first order Fourier coefficient suggests that the spin vectors of galaxies tend to be oriented perpendicular with respect to the equatorial coordinate system. Similarly, a positive value of first order Fourier coefficient suggests that the spin vectors of galaxies tend to be oriented parallel with respect to the equatorial coordinate system. Whereas, in the statistics of $\Phi$, a positive $\left(\Delta_{11} / \sigma\left(\Delta_{11}\right)\right.$ with significant value suggests that the spin vector projections of galaxies tend to point radially with respect to the center of the equatorial coordinate system. Similarly, a significant negative value of $\left(\Delta_{11} / \sigma\left(\Delta_{11}\right)\right.$ implies that the spin vector projection of galaxies tend to orient tangentially with respect to the equatorial coordinate system.

In addition to the statistical tests, we also study the 'humps' (bins with more solutions than the expected) and 'dips' (bins with less solutions than the expected) in the polar and azimuthal angle distributions. The solid curve, in the histogram of the $\theta$-distribution, represents the expected isotropic distribution whereas dashed curve is the cosine distribution. The solid circles with $\pm 1 \sigma$ error bars represent the observed distribution. The shaded portion represents the range $0^{\circ}<\theta<45^{\circ}$. A hump (or dip) in the smaller $\theta$ suggests that the spin vectors of galaxies tend to orient parallel (or perpendicular) with respect to the equatorial coordinate system.

Similarly, a hump (or dip) in the larger $\Phi$ indicates that the spin vectors of galaxies tend to be oriented perpendicular with respect to the equatorial coordinate system. In the histogram of the $\Phi$-distribution, solid curve represents the expected isotropic distribution whereas dashed curve is the average distribution. The solid circles with $\pm 1 \sigma$ error bars represent the observed distribution. The shaded portion represents the range $-45^{\circ}<\Phi<+45^{\circ}$. The humps and dips in the histograms of $\Phi$-distribution are not so easy to interpret as compared to $\theta$-distributions. It is because the range of $\Phi$ is $-90^{\circ}$ to $+90^{\circ}$. In the histogram of the $\Phi$-distribution, $\Phi=0^{\circ}$ means spin vector projections tend to point radially towards the center of the equatorial coordinate system. A hump in the middle (central eight bins) of the histogram suggests that the spin vector projections of galaxies tend to point towards the center of the chosen co-ordinate system. Similarly, a hump at first four and last four bins indicates that the spin vectors projections of galaxies tend to be oriented tangentially with respect to the chosen reference coordinate system.

Table 1: Statistics of the polar (left) and azimuthal angle (right) distributions of $r$-magnitude SDSS galaxies having redshift in the range 0.10 to 0.11.The $P\left(>\chi^{2}\right)$ represents the chi-square probability, $C / C(\sigma)$ represents the auto-correlation coefficient, $\left(\Delta_{11} / \sigma\left(\Delta_{11}\right)\right.$ is the first order Fourier Coefficient andP $\left(>\Delta_{1}\right)$ is the first order Fourier probability.

\begin{tabular}{|c|c|c|c|c|}
\hline Sample & $\mathrm{P}\left(>\chi^{2}\right)$ & $C / C(\sigma)$ & $\left(\Delta_{11} / \sigma\left(\Delta_{11}\right)\right.$ & $\mathrm{P}\left(>\Delta_{1}\right)$. \\
\hline r01 & 0.301 & -0.2 & 0.4 & 0.131 \\
\hline r02 & 0.901 & -0.5 & 1.8 & 0.911 \\
\hline r03 & 0.735 & 0.4 & 0.1 & 0.705 \\
\hline r04 & 0.423 & 0.7 & 0.2 & 0.323 \\
\hline r05 & 0.728 & 0.4 & -0.6 & 0.788 \\
\hline r06 & 0.912 & 0.3 & 1.5 & 0.992 \\
\hline r07 & 0.118 & -0.7 & -1.4 & 0.238 \\
\hline r08 & 0.115 & -0.8 & -1.3 & 0.105 \\
\hline r09 & 0.133 & -1.2 & 1.6 & 0.103 \\
\hline r10 & 0.828 & 1.3 & -1.2 & 0.828 \\
\hline r11 & 0.859 & -0.7 & 0.8 & 0.709 \\
\hline r12 & 0.802 & 0.8 & 0.9 & 0.082 \\
\hline r13 & 0.163 & 0.2 & 0.1 & 0.603 \\
\hline r14 & 0.464 & -0.5 & 0.6 & 0.44 \\
\hline & & & & \\
\hline
\end{tabular}

\begin{tabular}{|c|c|c|c|c|}
\hline Sample & $\mathrm{P}(>c 2)$ & $C / C(\sigma)$ & $\left(\Delta_{11} / \sigma\left(\Delta_{11}\right)\right.$ & $\mathrm{P}\left(>\Delta_{1}\right)$ \\
\hline $\mathrm{r} 01$ & 0.441 & -0.1 & 1.4 & 0.211 \\
\hline $\mathrm{r} 02$ & 0.891 & -0.3 & 1.2 & 0.202 \\
\hline $\mathrm{r} 03$ & 0.785 & 0.2 & 1.1 & 0.123 \\
\hline $\mathrm{r} 04$ & 0.243 & 0.5 & 0.5 & 0.444 \\
\hline $\mathrm{r} 05$ & 0.278 & 0.9 & -0.4 & 0.235 \\
\hline $\mathrm{r} 06$ & 0.892 & 0.1 & 1.3 & 0.556 \\
\hline $\mathrm{r} 07$ & 0.228 & -0.8 & -1.2 & 0.232 \\
\hline $\mathrm{r} 08$ & 0.225 & -0.2 & -1.4 & 0.232 \\
\hline $\mathrm{r} 09$ & 0.243 & 0.1 & 1.5 & 0.233 \\
\hline $\mathrm{r} 10$ & 0.858 & 2.1 & -1.1 & 0.204 \\
\hline $\mathrm{r} 11$ & 0.919 & -1.7 & 0.6 & 0.242 \\
\hline $\mathrm{r} 12$ & 0.882 & 1.8 & 0.3 & 0.112 \\
\hline $\mathrm{r} 13$ & 0.663 & 0.1 & 0.2 & 0.223 \\
\hline $\mathrm{r} 14$ & 0.654 & -0.2 & 0.7 & 0.114 \\
\hline & & & & \\
\hline
\end{tabular}

No preferred alignment is noticed in the spatial orientation of spin vectors of r-magnitude SDSS galaxies that have redshift in the range 0.10 to 0.11 , suggesting Hierarchy model. However, local effects are found in the samples $r 02$ and $r 09$.

In these samples, we suspect a local tidal connection between the rotation axes of galaxies or due to the merging process, the angular momentum vector of few galaxies get affected. A random alignment of spin vector projection ofall most galaxies is noticed in all samples suggesting Hierarchy model and supporting the better reliability of reference co-ordinate system. 

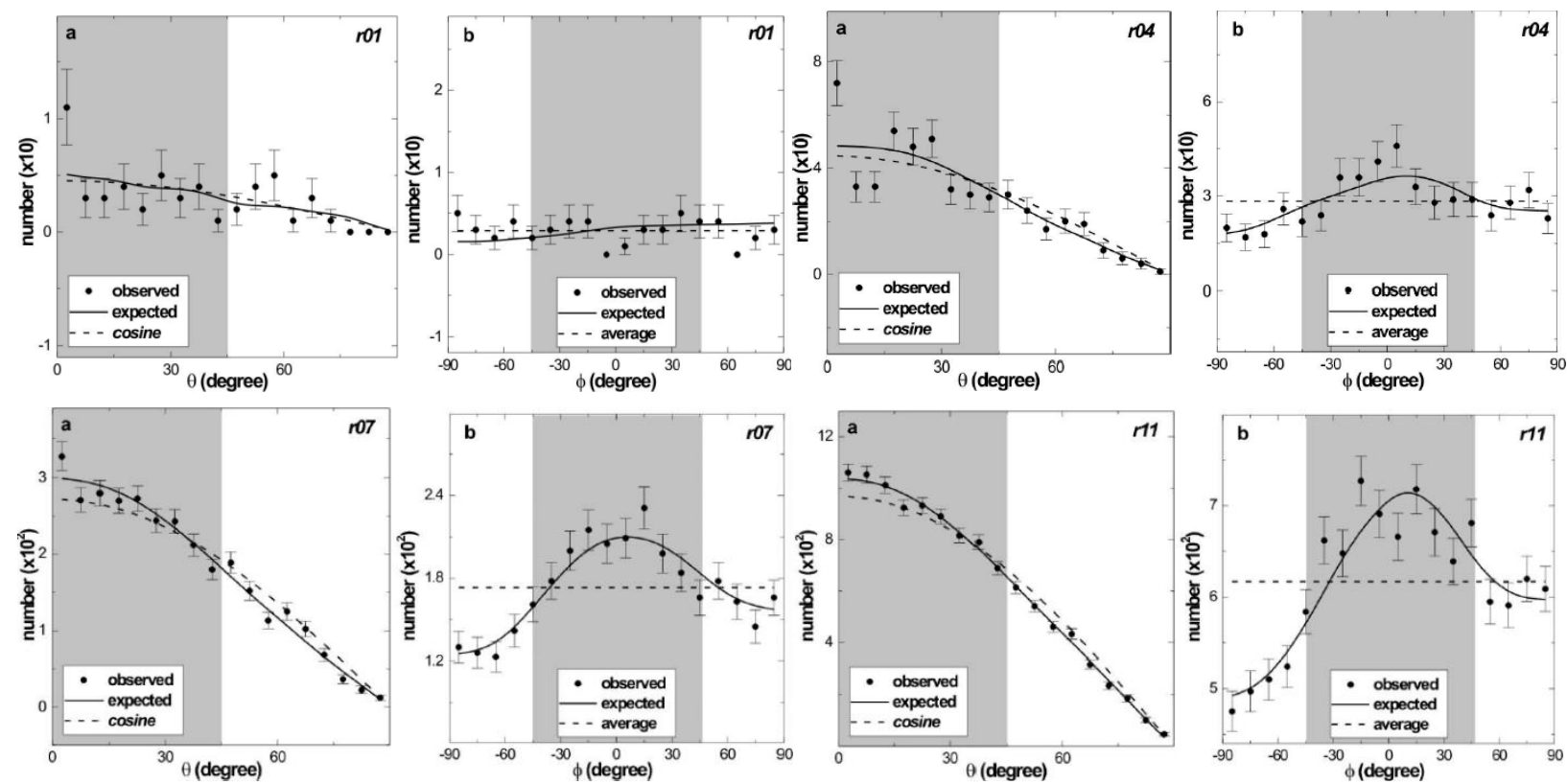

Figure 1: The polar $(\theta)$ and azimuthal angle $(\Phi)$ distributions of SDSS galaxies having $u$-magnitude. The solid line represents the expected isotropic distributions. The cosine and average distribution (dashed) is shown for the comparison. The statistical error $( \pm 1 \sigma)$ bars are shown for the observed counts.

\section{CONCLUSION}

We have studied the $r$-magnitude dependence in thepreferred alignment of spin vector orientation of 44,749 SDSSgalaxies that have redshift in the range 0.10 to 0.11 . We used the method proposed by Flin\&Godowsky ${ }^{8}$ in order to compute two-dimensional data to three dimensional galaxyrotation axis (polar \& azimuthal angles). We have carried outrandom simulation by creating $10^{7}$ virtual galaxies andadopting the method proposed by Aryal\&Saurer ${ }^{11}$ in order to find theoretical distribution of galaxy rotation axes. We have compared the differences between theoretical distributions and observed distributions using three statistics, namely chisquare, auto-correlation and the Fourier. We conclude our results as follows:

1. No preferred alignment is noticed in the spatial orientation of spin vectors of galaxies, suggesting Hierarchy model, ${ }^{5}$. However, a local effect is noticed in the samples $r 02$ and $r 09$. In these samples, we suspect a local tidal connection.

2. In the deep field, density fluctuation is expected and observed in the local scale. Existence of Superclusters in the region of interest cannot be ruled out.

3. We have used equatorial system as a physical reference in order to study non-random effects concerning galaxy orientation. Hierarchy model predicts that the choice of co-ordinate system donot alter preferred alignments. However, pancake model advocates the importance of physical reference system. We found mixed results: in most cases Hierarchy model and in a few cases Pancake model. This problem should be addressed by using another system such as Galactic or Super galactic systems.

4. A random simulation for $10^{7}$ virtual particles (i.e, galaxies) carried out successfully by using 4 GB RAM pc. We intend to carry out simulation generating $10^{8}$ virtual galaxies in order to find out sufficiently smooth expected isotropic distribution curves in the future.
In general, our results support Hierarchy model. The spatial orientation of galaxies in 117 clusters were studied

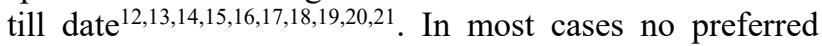
alignment is noticed (Hierarchy model). However, few clusters strongly support Pancake model. Aryal et al noticed a systematic change in the galaxy alignments from early-type (BM I) to late-type (BM III) clusters ${ }^{[19]}$.

\section{ACKNOWLEDGEMENTS}

This research has made use of the SDSS database. We acknowledge Prof. Binil Aryal for critical discussions. We thank Mr. Rajesh Kumar Bachchan for his help during data compilation and Mr. Ajay Kumar Jha for his very excellent suggestion.

\section{REFERENCES}

1. Gamow, G. 1952. Phys. Rev. 86:251.

2. Doroshkevich, A. G. 1970. Astrophysics.6:320.

3. Doroshkevich, A. G. 1973.Astrophysical Journ. 14: L11

4. Doroshkevich, A. G. and Shandarin, S. F. 1978. Monthly Notices Royal Astron. Soc. 184: 643.

5. Peebles, P. J. E.1969. Astrophysical, Journ. 155: 393.

6. Ozernoy, L. M. in Longair M. S. J., eds, 1978. Proc. IAUSymp. 79, Reidel, Dordrecht.

7. Stein, R. 1974.Astron\&Astrophys. 35: 17

8. Flin, P. and Godlowski W. 1986. Monthly Notices Royal Astron. Soc. 222:525

9. Holmberg, E.Medd. Lund. Astron. Obs, Ser. VI, No. 117:1946.

10. Heidmann, J.Heidmann, N. and deVaucouleurs, G. 1971. Monthly Notices Royal Astron. Soc. 75: 85.

11. Aryal, B. and Saurer, W. 2000.AstronomAstrophys let. 364:L97.

12. Godlowski, W. 1993. Monthly Notices Royal Astron. Soc. 265: 874.

13. Godlowski, W. 1994. Monthly Notices Royal Astron. Soc. 271: 19.

14. Flin, P. 2001. Monthly Notices Royal Astron. Soc. 325: 49.

15. Aryal, B. Bhattarai ,H. Rajbahak C. \& Saurer W., 2013, Monthly Notice of Royal Astron. Soc. 434: 1939.

16. Aryal B., Yadav S. N. and Saurer, W. 2012. Bulletin of Astron. Astron. Soc. Ind. (BASI), 40:65.

17. Aryal, B. Paudel, S. and Saurer, W. 2008.Astronom. Astrophys.479: 397

18. Aryal, B. and Saurer, W. 2006. Monthly Notices Royal Astron. Soc. 366:438

19. Aryal, B. and Saurer, W. 2005a.Astronom. Astrophys.432: 841

20. Aryal, B. and Saurer, W. 2005b.Astronom. Astrophys.432:431.

21. Aryal, B. and Saurer, W. 2004.Astronom. Astrophys.425:871. 